\title{
EVENTRATION OF THE DIAPHRAGM*
}

BY

\author{
ERIC GOULSTON \\ From the Royal Alexandra Hospital for Children, Sydney
}

This term is accepted as meaning marked elevation of all or part of one hemi-diaphragm due to a congenital aberration in development. Its complex embryonic formation subjects this musculo-fascial structure to areas of potential weakness which is aggravated by the strain of a relative negative pressure in the thorax and the inspiratory muscular pull. Wrong timing, failure of adequate fusion and failures of development lead to weak points and there are therefore opportunities for variation in size and shape as well as for defects.

A congenital hypoplasia with non-development of muscular elements is accepted as its causation leaving the involved diaphragm a mere fibrous sheet or atrophic membrane containing few or no muscular fibres in its peripheral rim. The hemi-diaphragm may function paradoxically if there is also faulty phrenic innervation. The stomach angulates upwards, compressing the lung and displacing the heart and mediastinum, and may cause cardio-respiratory embarrassment. Eventration may be total, which is more frequent on the left, or partial, which is commoner on the right side where the appearance may be mistaken for an accessory liver lobe. It is sometimes associated with arthrogyposis congenita. The incidence is said to be 1 in 10,000 of births.

There may be no symptoms or varying degrees of dyspnoea and cyanosis and these are referable to the cardiovascular, respiratory or digestive systems.

The diagnosis is usually established by radiology revealing an abnormally high position of the affected hemi-diaphragm with a smooth, unbroken arc, and the heart and mediastinum displaced toward the normal side. As diaphragmatic movement is due to varying pressure in the thorax and abdomen as well as the muscle itself, one may see normal motion, but usually fluoroscopy reveals lessened respiratory excursion and occasionally paradoxical movement. Barium studies may help in differentiating the condition from a diaphragmatic hernia where the diaphragm is more difficult to outline and pneumoperitoneal studies may be of value.

* A paper read at the meeting of the British Association of Paediatric Surgeons in London in July 1956.
Complications, while rare, do occur, and rupture of the thinned-out portion of the diaphragm during labour has been reported. Peptic ulceration, volvulus of the stomach and pneumonia from inadequate pulmonary expansion may occur.

Treatment varies according to the severity of the symptoms and must be as prompt in the newborn with cyanosis and dyspnoea as when caused by a large diaphragmatic hernia. When indicated by cardiovascular or respiratory distress, surgical intervention by the thoracic, abdominal or thoracoabdominal approach is made. The diaphragm is repaired by plication or overlapping with interrupted non-absorbable sutures using tantalum or nylon mesh reinforcement if necessary.

Complete eventration has been repaired in the newborn by suturing the anterior portion of the diaphragm to the circumference of the costal margin of the diaphragm anteriorly, and the posterior portion sewn to the crus medially and renal fascia laterally. The liver in these cases has no peritoneal attachment to the diaphragm and is easily mobilized. If, after replacement, the abdomen is found to be too tense, only the abdominal skin wound is sutured as described by Gross for diaphragmatic hernia.

During the past five years five patients have been admitted to the Royal Alexandra Hospital, Sydney, with a diagnosis of eventration of the diaphragm.

\section{Case Reports}

Case 1. N.H., aged 5 days, was admitted to the Royal Alexandra Hospital for Children in 1951 with a history of grunting respirations and repeated cyanotic attacks. After admission the baby's condition remained good, there was no cyanosis or distress and after three weeks' observation he was discharged. The chest radiograph showed the left hemi-diaphragm markedly elevated, with the heart and mediastinum displaced to the right. The left dome moved evenly with the right. He has remained well since. A chest radiograph in 1955, when he was still symptom-free, showed marked elevation of the left hemi-diaphragm with the heart and mediastinum still displaced to the right side.

Case 2. K.B. was admitted in 1954 aged 3 weeks, with a history of cyanosis except when oxygen was administered and rapid respirations. A caesarean operation had 
been performed on account of a shoulder presentation. After admission, the baby appeared to be in no distress. A chest radiograph revealed an elevated right hemidiaphragm which moved paradoxically suggesting phrenic paralysis. A barium study showed the distal end of the stomach much higher than the cardia due to elevation of the liver. This baby was discharged after two weeks in hospital and has remained symptom free since.

Case 3. D.F., aged 6 years, was admitted in 1953 with a history of chronic cough and recurring attacks of vomiting since birth. A chest radiograph showed the anterior two-thirds of the left hemi-diaphragm elevated and this portion exhibited paradoxical movement. The posterior third was normally attached and contracted normally with the right side. The heart was displaced to the right, and the stomach rotated anti-clockwise. There was scoliosis convex to the left. A thoracotomy operation was performed and the redundant portion of the thin fibrous central portion of the diaphragm excised with suture of the muscular margins. Histologically the tissue examined consisted entirely of compact acellular bands of collagen and was devoid of any striated muscle. This girl continued to vomit, especially after lying down at night, and a subsequent chest radiography showed a similar bulge of the left hemi-diaphragm. Thoracotomy was repeated later and the defect closed by a fascia lata reinforcement. She has been well since.

Case 4. J.H., aged 7 years, was operated upon two years ago for repeated episodes of bronchitis, with a chest radiograph showing a large eventration in the left hemidiaphragm. At thoracotomy the central membranous area of the diaphragm was excised, sutured at the margins and reinforced. Histologically the specimen consisted of collagen tissue with no striated muscle. He has been well since.

Case 5. A man, aged 48 years, complained of pain in the left chest for 2 months aggravated by coughing, and vomiting and dyspnoea for 4 days. There was no history of trauma or any earlier relevant symptoms. He is a zoo attendant and says he has to do heavy lifting at times.
On admission to hospital, he was ill, dyspnoeic and distressed. There was no movement in the left chest, which showed signs of pleural effusion. A chest radiograph reported an eventration of the diaphragm with mediastinal shift and a large amount of stomach in the left chest. A barium swallow showed marked elevation of the left diaphragm with paradoxical movement and volvulus of the stomach. Great relief was obtained by gastric suction, and at a subsequent operation a 3 in. rent was found in a thinned central portion of the left diaphragm through which stomach, spleen and splenic colon had entered. These organs were reduced and the rent plicated securely. Convalescence and subsequent progress was uneventful. This appears to be a rare case of rupture of a partial eventration on the left side. $\mathrm{Mr}$. Norman Tanner said recently that he had encountered five or six cases since 1943 of volvulus of the stomach in adults secondary to eventration of the diaphragm. He merely ligates the gastro-colic omentum and allows the colon to go up into the chest and places a few anchoring sutures in the stomach bed.

Case 6. A baby aged 3 months was admitted on account of frequent vomiting and cyanotic attacks but died soon after admission to hospital. The necropsy revealed faulty development of the diaphragm on each side so that the central portions were formed of membrane only, while the muscle arched anteriorly and posteriorly around the area of muscular deficiency. On the right side the right lobe of the liver projected into and occupied one half of the pleural cavity. On the left side the spleen and distended stomach projected upwards similarly. None of the viscera were within the pleural sac as the membranous diaphragm enclosed them above. The lungs were malformed and collapsed and directly related to the misplaced abdominal viscera. The liver was misshaped and deeply grooved.

\section{Summary}

A résumé of eventration of the diaphragm is given. Case histories of five infants and one adult with rupture are described. 